Case Report

\title{
Infrarenal Infected Aortic Aneurysm Caused by Streptococcus pyogenes
}

\author{
Floryn Cherbanyk, ${ }^{1}$ Markus Menth, ${ }^{1}$ Bernhard Egger, ${ }^{1}$ and Véronique Erard ${ }^{2}$ \\ ${ }^{1}$ Department of General Surgery, HFR Fribourg-Cantonal Hospital, 1708 Fribourg, Switzerland \\ ${ }^{2}$ Department of Medicine, HFR Fribourg-Cantonal Hospital, 1708 Fribourg, Switzerland \\ Correspondence should be addressed to Bernhard Egger; bernhard.egger@h-fr.ch
}

Received 25 November 2016; Accepted 28 February 2017; Published 19 April 2017

Academic Editor: Einar Dregelid

Copyright (C) 2017 Floryn Cherbanyk et al. This is an open access article distributed under the Creative Commons Attribution License, which permits unrestricted use, distribution, and reproduction in any medium, provided the original work is properly cited.

Infectious aneurysm is a rare entity associated with significant morbidity and mortality. Current knowledge on pathogenesis, outcome, diagnosis, management, and follow-up remains debatable. We report the case of a patient with Streptococcus pyogenes aneurysm who was successfully treated with a homograft implant and discuss microbiological characteristics, diagnostic methods, and treatment options currently available for this serious disease.

\section{Introduction}

Infectious aneurysm, commonly called "mycotic aneurysm," refers to any aneurysm that result from bacterial and fungal infections of the arterial wall $[1,2]$. Before the antibiotic era and improvements in the field of cardiac surgery, infectious aneurysms were usually secondary to infectious endocarditis. Nowadays, atherosclerosis predominantly affecting the aorta and iliofemoral arteries (and its determinants: increasing age, smoking, and male gender) and immunosuppression are the principal predisposing factors of the disease [3]. Bacteria including Staphylococcus aureus, Streptococcus pneumonia, Streptococcus viridans, and various Enterobacteriaceae especially nontyphi Salmonella species are the usual pathogens [4]. Given the lack of conclusive signs and symptoms, patients are subjected to various investigative modalities, until a diagnosis is reached. Antibiotics and open surgery are the standard treatment; however, endovascular repair is also an option. To date, there is no consensus on the best approach.

\section{Case Presentation}

A 69-year-old woman was admitted with a 3-week history of recurrent abdominal pain and intermittent fever up to $39^{\circ} \mathrm{C}$. First manifestations occurred about 10 days after she had taken care of her grandchildren, who at the time they were being treated for scarlet fever. In her medical history, she had hysterectomy, bilateral adnexectomy, cholecystectomy, appendectomy, and sigmoïdectomy for diverticulitis.

On admission, the clinical status was relevant only by a diffuse painful abdominal palpation and a temperature of $38,5^{\circ} \mathrm{C}$. Blood pressure, pulse rate, and saturation were $115 / 55 \mathrm{mmHg}, 89 / \mathrm{min}$, and $96 \%$. Femoral and peripheral pulses were palpable.

At admission, C-reactive protein (CRP) was $67 \mathrm{mg} / \mathrm{l}$ (normal value $<5 \mathrm{mg} / \mathrm{l}$ ) and leukocyte count was $15.2 \mathrm{G} / \mathrm{l}$ (normal value 4.0-10 G/l). Abdominal CT scan showed a circumferential thickening of the sigmoid wall, without fat infiltration, and poorly delimitated infrarenal periaortic infiltrate in contact with the inferior vena cava (Figure 1).

A presumptive diagnosis of intestinal infection was made. Piperacillin-tazobactam was initiated. All four blood cultures that were performed were positive for Streptococcus pyogenes, causing the initial diagnosis to be reconsidered. A second look of the first CT scan revealed possible signs of an aortic process. A second CT scan on day 6 showed an increased periaortic infiltration highly suggestive of infectious aortitis (Figure 2). Transesophageal echocardiography excluded endocarditis. 


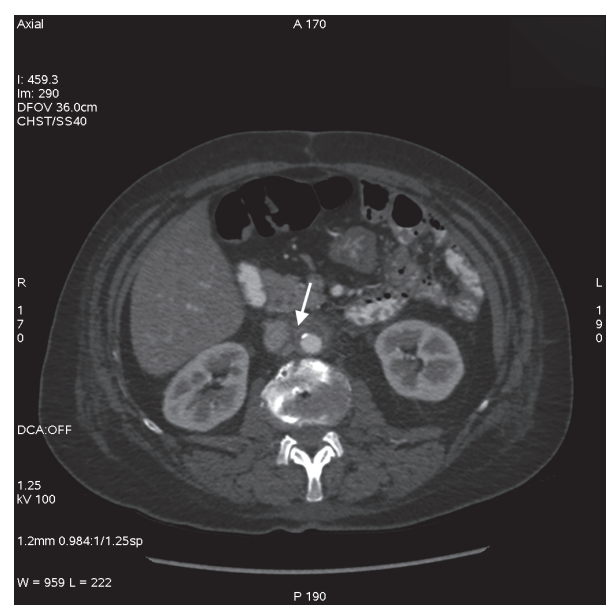

FIGURE 1: Abdominal CT scan (day 1) revealing infrarenal periaortic infiltrates in contact with the inferior vena cava (arrow).

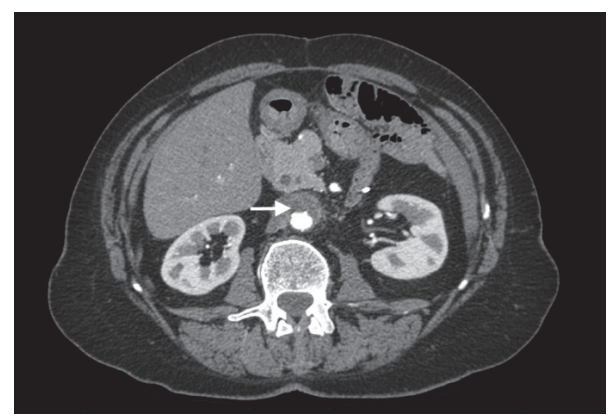

FIgURE 2: Abdominal CT scan (day 6) showing an increased periaortic infiltration highly suggestive of infectious aortitis (arrow).

Piperacillin-tazobactam was replaced with ceftriaxone. The patient was referred to a cardiovascular surgery reference center. Vascular surgery was initially not considered. However, one month after admission and despite favorable clinical and biological evolution, the patient experienced severe abdominal pain. The angio-CT scan displayed an infrarenal aortic aneurysm about to rupture (Figures 3(a) and 3(b)), prompting urgent open abdominal surgery. A xiphopubic laparotomy was performed, revealing an infrarenal aneurysm with signs of chronic inflammation. No pus was present externally. After clamping the aorta immediately distally of the left renal artery, as well as the two iliac arteries, the aneurysm was opened longitudinally. The inside contained thrombotic material that was sent for pathological and microbiological examination. The aneurysm was then completely excised and a thoracic homograft was put in place (Figure 4) and covered with porcine pericardium. Cultures of the operative samples remained negative.

Antibiotic therapy with ceftriaxone was continued for two weeks postoperatively and then replaced with oral Clindamycin for 8 additional weeks. The patient made an uneventful recovery and medical and radiological examinations (CT scan) performed at months 6, 12, 24, 72, and 84 after surgery were unremarkable.

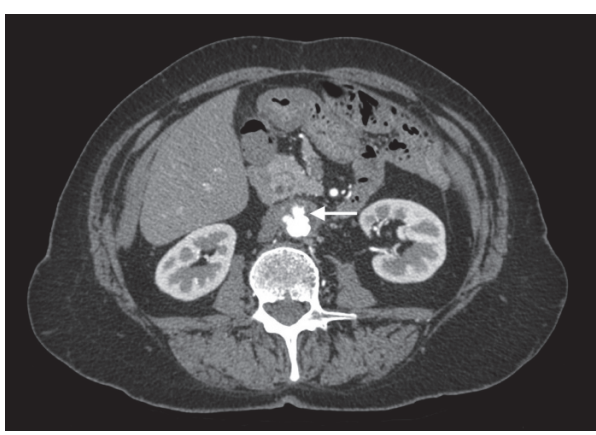

(a)

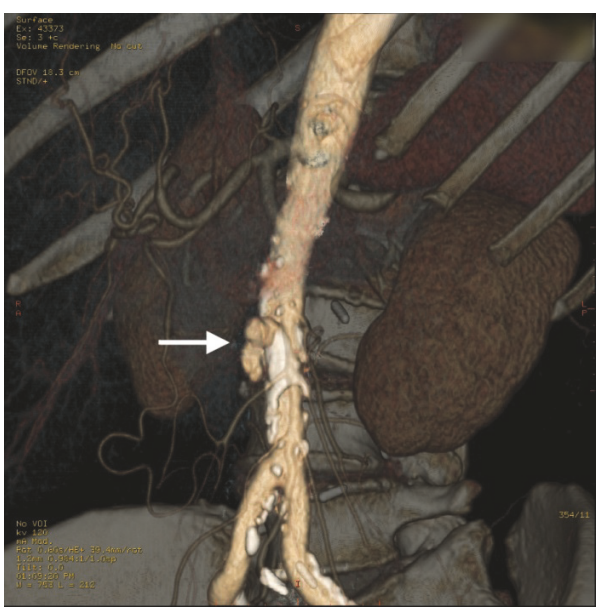

(b)

FIgURE 3: Axial (a) and reconstructed computed tomographic angiography (b) showing signs of prerupture.

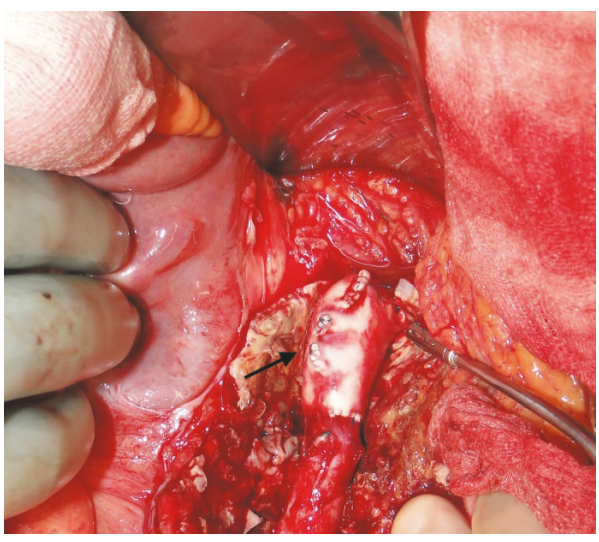

FIGURE 4: Intraoperative view showing the infrarenal aortic replacement with a homograft (black arrow).

\section{Discussion}

Infectious aneurysm is a rare entity, representing less than $2 \%$ of all aortic aneurysms [5]. The aorta is normally very resistant to infection, but conditions such as atherosclerosis, malformation, arteriovenous fistula, and bacterial invasion of the vasa vasorum may lead to arteritis and therefore 
infectious aneurism formation [6-8]. In addition, diabetes, chronic renal failure, chronic steroid exposition, or some degree of immunosuppression can contribute to the development arteritis.

Prior to the antibiotic era, infectious aortitis was commonly associated with bacterial endocarditis involving mostly S. viridans, S. pneumoniae, and Haemophilus influenzae [9]. Nowadays, the majority of reported organisms seen in infectious aneurysm are staphylococcal species, nonhaemolytic types of Streptococci, and Salmonella species $[10,11]$. S. pyogenes is responsible for a diverse range of infectious pathologies but is only exceptionally associated with endovascular infection. In 2013, Gardiner et al. reported one case of $S$. pyogenes aortic aneurysm occurring 4 weeks after an episode of febrile pharyngitis and managed to collect only 8 additional cases from the literature (see Table 1) [12]. In our patient, the only infectious source identified was a prolonged contact with her grandchildren diagnosed with systemic streptococcal infection.

The clinical presentation of infected aortitis is often subtle with a lack of conclusive signs or symptoms. A high index of suspicion is therefore needed in order to reach the diagnosis in the presence of fever, abdominal pain, and/or positive blood cultures of undetermined cause. A significant proportion of blood or tissue cultures remains negative ranging, respectively, from $25-37 \%$ and $22-50 \%[4,13,14]$. Once the diagnosis is suspected, CT angiography remains the investigative modality of choice, although CT alone or MRI with gadolinium is also adequate in establishing the diagnosis of infectious aneurysm and evaluating the degree of emergency $[3,15,16]$.

Infectious aneurysms is still associated with a high mortality rate in spite of advanced antibiotic therapy and improved surgical technic. It is established that the standard treatment of infectious aneurysm is a combination of antibiotics and surgery. To date, there, however, is still no consensus on specific management and the timing and type of surgery as well as antibiotic therapy duration remain debated [17]. Surgical treatment is dependent on numerous factors, including clinical and radiological status, localization of infection, antibiotic treatment prior to surgery, and type of bacteria. Surgery should be delayed if possible, as the outcome for patients with elective surgery seems more favorable than that of patients who require emergency surgery [18].

The standard surgical treatment for infrarenal infectious aneurysm consists of aneurysmectomy, extensive debridement of the surrounding soft tissue, and revascularization $[10,19,20]$. Options for revascularization of infrarenal aorta include in situ reconstruction with use of prosthetic graft or homograft, as well as extra-anatomic bypass and endovascular stent graft [12, 21-23]. The extra-anatomical axillofemoral bypass used to be considered as the standard surgical procedure for infrarenal infectious aneurysms, but outcomes are far from ideal and the magnitude and long duration of the operation compromise its feasibility in severely ill patients. Furthermore, bypass thrombosis is reported in up to $25 \%$ of cases despite anticoagulation, and reinfection of the bypass has been reported to be as high as $40 \%[24,25]$. Finally, hemorrhage of the aortic stump is described in up to $20 \%$ of cases $[8,26,27]$. In situ reconstruction with use of a prosthetic graft is attended by a greater risk of graft infection than extra-anatomical bypass procedure. Compared to synthetic grafts, the use of cryopreserved grafts has the advantage of low vulnerability to infection and limited immune response with better viability $[28,29]$. This procedure is limited by its cost and the fact that it is not applicable in urgent situations because it needs to be ordered from an international graft banking center. Furthermore, cryopreserved grafts can become dilated on the long term and chronic rejection can lead to local thrombus formation [21]. Reports of open repair of infectious aneurysm indicate a mortality of more than $20 \%$ with significant short- and long-term morbidity related to the operation [30], precluding its use in severely ill or shocked patient. Endovascular stent grafts have been introduced as an alternative permitting minimally invasive interventions, prompt aneurysm exclusion, and immediate control of bleeding [31-33]. However, to date, the consensus of expert opinion does not favor the endovascular repair and it is usually reserved for patients with prohibitively high risk for open surgical repair [32]. This restriction is mainly due to the fact that endovascular technique precludes effective drainage of suppuration and debridement of infected tissues. In any case, prosthesis in infected sites can still be used as a temporary treatment prior to definitive open surgical repair $[31,34,35]$. For all these reasons, it is obvious that treatment options need to be evaluated case by case by a multidisciplinary team.

Long-term antibiotics are always indicated; however, their use and duration postoperatively have not been extensively studied [14]. It is reasonable to administer antibiotics for several days or even weeks prior to surgery as long as the clinical situation is stable [36]. In urgent settings, empirical treatment should include large spectrum antibiotics active on Gram-negative Enterobacteriaceae and Gram-positive bacteria, started preoperatively after taking blood cultures. In the absence of positive cultures, molecular diagnostic tests (PCR detection) may help to identify the organisms and determine an adequate postoperative antibiotic treatment. The duration of antibiotic treatment after surgery is not established precisely, ranging from 6 weeks to lifelong therapy $[19,37]$. In our case, the length of antibiotic therapy was decided based on the clinical evolution as well as laboratory and radiological results.

Infectious aneurysm is therefore a challenging disease regarding its diagnosis and its management and needs a multidisciplinary plan of treatment.

\section{Conclusion}

Untreated infectious arteritis ultimately leads to aneurysm. Because the clinical signs and symptoms are subtle and unspecific, diagnosis is often delayed until the disease has reached an advanced stage. Elective surgery is preferable to an emergency intervention. Antibiotics are a crucial factor of success and should be initiated as soon as possible; however, there is no universal recommendation regarding postoperative antibiotic treatment. The surgical technique of 


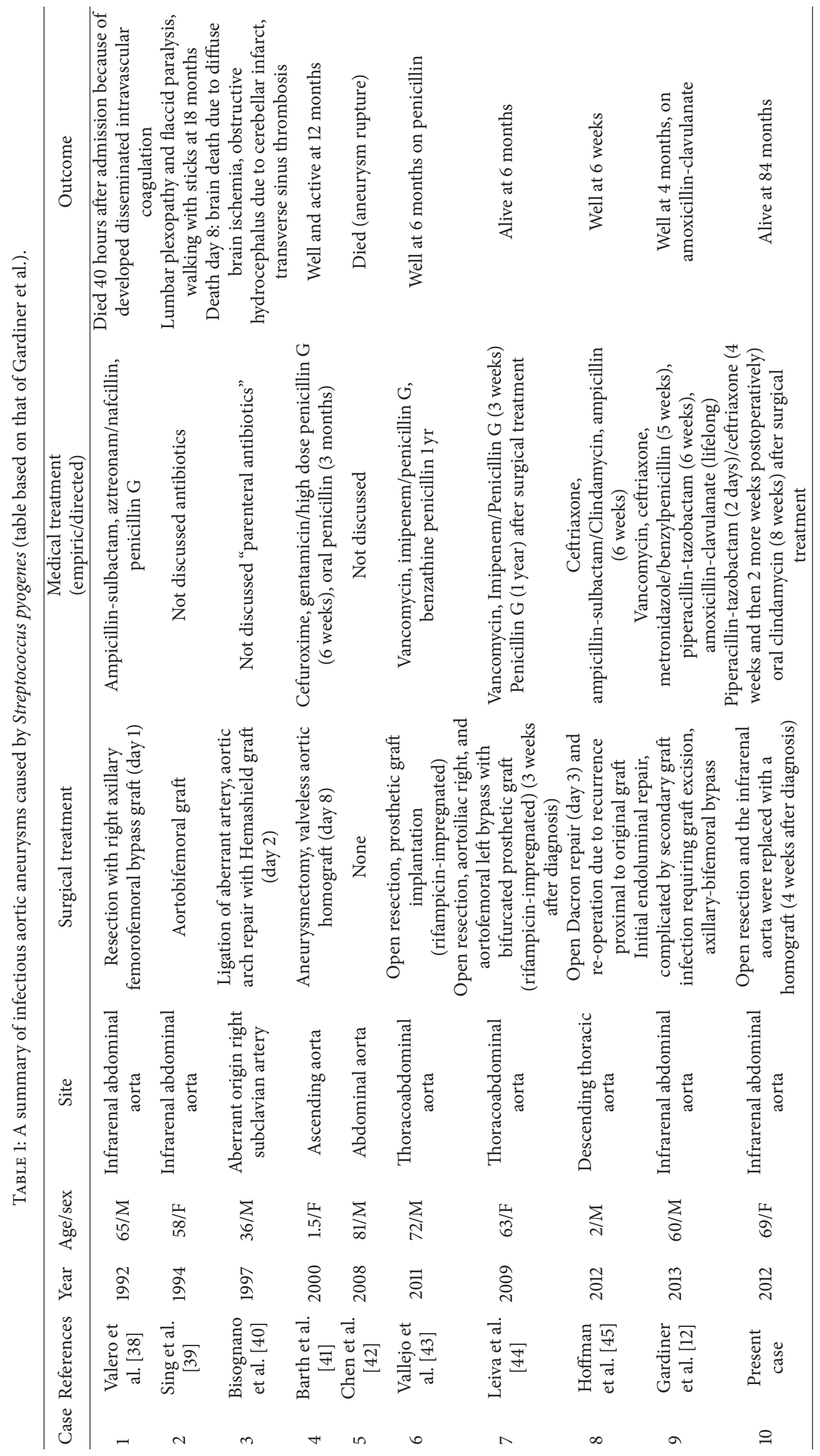


choice remains disputed, with endovascular repair gaining in popularity but remaining problematic because of graft infection.

\section{Conflicts of Interest}

The authors declare no conflicts of interest.

\section{References}

[1] S. E. Wilson, P. V. Wagenen, and E. Passaro Jr., "Arterial infection," Current Problems in Surgery, vol. 15, no. 9, pp. 1-89, 1978.

[2] V. Parameswaran, "Multiple mycotic aneurysms with a rare fungus, Aspergillus niger: a complex case report," Journal of Vascular Nursing, vol. 26, no. 1, pp. 22-26, 2008.

[3] H. L. Gornik and M. A. Creager, "Aortitis," Circulation, vol. 117, no. 23, pp. 3039-3051, 2008.

[4] D. V. Miller, G. S. Oderich, M.-C. Aubry, J. M. Panneton, and W. D. Edwards, "Surgical pathology of infected aneurysms of the descending thoracic and abdominal aorta: clinicopathologic correlations in 29 cases (1976 to 1999)," Human Pathology, vol. 35, no. 9, pp. 1112-1120, 2004.

[5] G. S. Oderich, J. M. Panneton, T. C. Bower et al., "Infected aortic aneurysms: aggressive presentation, complicated early outcome, but durable results," Journal of Vascular Surgery, vol. 34, no. 5, pp. 900-908, 2001.

[6] M. N. Gomes, P. L. Choyke, and R. B. Wallace, "Infected aortic aneurysms: a changing entity," Annals of Surgery, vol. 215, no. 5, pp. 435-442, 1992.

[7] E. A. Foote, R. G. Postier, R. A. Greenfield, and M. S. Bronze, "Infectious aortitis," Current Treatment Options in Cardiovascular Medicine, vol. 7, no. 2, pp. 89-97, 2005.

[8] M. C. Oz, B. J. Brener, J. A. Buda et al., "A ten-year experience with bacterial aortitis," Journal of Vascular Surgery, vol. 10, no. 4, pp. 439-449, 1989.

[9] A. Stengel and C. C. Wolferth, "Mycotic (bacterial) aneurysms of intravascular origin," Archives of Internal Medicine, vol. 31, no. 4, pp. 527-554, 1923.

[10] M. Dubois, K. Daenens, S. Houthoofd, W. E. Peetermans, and I. Fourneau, "Treatment of mycotic aneurysms with involvement of the abdominal aorta: single-centre experience in 44 consecutive cases," European Journal of Vascular and Endovascular Surgery, vol. 40, no. 4, pp. 450-456, 2010.

[11] M. V. Martins Cury, M. H. De Campos, and D. P. Dos Santos, "Salmonella-related mycotic pseudoaneurysm of the superficial femoral artery," International Journal of Surgery Case Reports, vol. 3, no. 1, pp. 27-29, 2012.

[12] B. J. Gardiner, J. Wong, M. Yii et al., "Streptococcus pyogenes aortic aneurysm infection: forgotten but not gone," Infectious Disease Reports, vol. 5, no. 2, article el1, 2013.

[13] P.-J. Hsu, C.-H. Lee, F.-Y. Lee, and J.-W. Liu, "Clinical and microbiological characteristics of mycotic aneurysms in a medical center in southern Taiwan," Journal of Microbiology, Immunology and Infection, vol. 41, no. 4, pp. 318-324, 2008.

[14] K. Laohapensang, S. Aworn, S. Orrapin, and R. B. Rutherford, "Management of the infected aortoiliac aneurysms," Annals of Vascular Diseases, vol. 5, no. 3, pp. 334-341, 2012.

[15] R. H. Blair, M. D. Resnik, and J. P. Polga, "CT appearance of mycotic abdominal aortic aneurysms," Journal of Computer Assisted Tomography, vol. 13, no. 1, pp. 101-104, 1989.
[16] C. S. Restrepo, D. Ocazionez, R. Suri, and D. Vargas, "Aortitis: imaging spectrum of the infectious and inflammatory conditions of the aorta," Radiographics, vol. 31, no. 2, pp. 435-451, 2011.

[17] P.-L. Chen, L.-M. Tsai, C.-D. Kan, and W.-C. Ko, "Is 2 weeks of antibiotic therapy enough to treat elderly patients with nontyphoid Salmonella bacteremia? A case report of fatal endovascular infection," Journal of Microbiology, Immunology and Infection, vol. 47, no. 4, pp. 350-353, 2014.

[18] C.-D. Kan, H.-L. Lee, C.-Y. Luo, and Y.-J. Yang, “The efficacy of aortic stent grafts in the management of mycotic abdominal aortic aneurysm-institute case management with systemic literature comparison," Annals of Vascular Surgery, vol. 24, no. 4, pp. 433-440, 2010.

[19] R.-B. Hsu, Y.-G. Tsay, S.-S. Wang, and S.-H. Chu, "Surgical treatment for primary infected aneurysm of the descending thoracic aorta, abdominal aorta, and iliac arteries," Journal of Vascular Surgery, vol. 36, no. 4, pp. 746-750, 2002.

[20] B. T. Weis-Müller, C. Rascanu, A. Sagban, K. Grabitz, E. Godehardt, and W. Sandmann, "Single-center experience with open surgical treatment of 36 infected aneurysms of the thoracic, thoracoabdominal, and abdominal aorta," Annals of Vascular Surgery, vol. 25, no. 8, pp. 1020-1025, 2011.

[21] E. Kieffer, A. Bahnini, F. Koskas, C. Ruotolo, D. Le Blevec, and D. Plissonnier, "In situ allograft replacement of infected infrarenal aortic prosthetic grafts: results in forty-three patients," Journal of Vascular Surgery, vol. 17, no. 2, pp. 349-356, 1993.

[22] T. A. Koeppel, J. Gahlen, S. Diehl, R. L. Prosst, and C. Dueber, "Mycotic aneurysm of the abdominal aorta with retroperitoneal abscess: successful endovascular repair," Journal of Vascular Surgery, vol. 40, no. 1, pp. 164-166, 2004.

[23] E. D. Mundth, R. C. Darling, R. H. Alvarado, M. J. Buckley, R. R. Linton, and W. G. Austen, "Surgical management of mycotic aneurysms and the complications of infection in vascular reconstructive surgery," The American Journal of Surgery, vol. 117, no. 4, pp. 460-470, 1969.

[24] A. R. Naylor, S. Clark, N. J. M. London et al., "Treatment of major aortic graft infection: preliminary experience with total graft excision and in situ replacement with a rifampicin bonded prosthesis," European Journal of Vascular and Endovascular Surgery, vol. 9, no. 2, pp. 252-256, 1995.

[25] K. Johansen and J. Devin, "Mycotic aortic aneurysms: a reappraisal," Archives of Surgery, vol. 118, no. 5, pp. 583-588, 1983.

[26] J. M. Ewart, M. L. Burke, and T. J. Bunt, "Spontaneous abdominal aortic infections. Essentials of diagnosis and management," The American Surgeon, vol. 49, no. 1, pp. 37-50, 1983.

[27] M. Pasic, T. Carrel, M. Vogt, L. von Segesser, and M. Turina, "Treatment of mycotic aneurysm of the aorta and its branches: the location determines the operative technique," European Journal of Vascular Surgery, vol. 6, no. 4, pp. 419-423, 1992.

[28] T. Fischlein, A. Schütz, M. Haushofer et al., "Immunologic reaction and viability of cryopreserved homografts," The Annals of Thoracic Surgery, vol. 60, no. 2, supplement 2, pp. S122-S126, 1995.

[29] P. R. Vogt, H.-P. Brunner-LaRocca, M. Lachat, C. Ruef, and M. I. Turina, "Technical details with the use of cryopreserved arterial allografts for aortic infection: influence on early and midterm mortality," Journal of Vascular Surgery, vol. 35, no. 1, pp. 80-86, 2002.

[30] J. M. Fichelle, G. Tabet, P. Cormier et al., "Infected infrarenal aortic aneurysms: when is in situ reconstruction safe?" Journal of Vascular Surgery, vol. 17, no. 4, pp. 635-645, 1993. 
[31] K. Sörelius, K. Mani, M. Björck, R. Nyman, and A. Wanhainen, "Endovascular repair of mycotic aortic aneurysms," Journal of Vascular Surgery, vol. 50, no. 2, pp. 269-274, 2009.

[32] L. G. Svensson, N. T. Kouchoukos, D. C. Miller et al., "Expert consensus document on the treatment of descending thoracic aortic disease using endovascular stent-grafts," Annals of Thoracic Surgery, vol. 85, supplement 1, pp. S1-S41, 2008.

[33] Y.-K. Huang, C.-L. Chen, M.-S. Lu et al., "Clinical, microbiologic, and outcome analysis of mycotic aortic aneurysm: the role of endovascular repair," Surgical Infections, vol. 15, no. 3, pp. 290-298, 2014.

[34] C.-D. Kan, H.-L. Lee, and Y.-J. Yang, "Outcome after endovascular stent graft treatment for mycotic aortic aneurysm: a systematic review," Journal of Vascular Surgery, vol. 46, no. 5, pp. 906-912, 2007.

[35] K. G. Jones, R. E. Bell, T. Sabharwal, M. Aukett, J. F. Reidy, and P. R. Taylor, "Treatment of mycotic aortic aneurysms with endoluminal grafts," European Journal of Vascular and Endovascular Surgery, vol. 29, no. 2, pp. 139-144, 2005.

[36] R. J. Lopes, J. Almeida, P. J. Dias, P. Pinho, and M. J. Maciel, "Infectious thoracic aortitis: a literature review," Clinical Cardiology, vol. 32, no. 9, pp. 488-490, 2009.

[37] R. C. Jones, V. Reddy, L. Kornstein et al., "Salmonella enterica serotype Uganda infection in New York City and Chicago," Emerging Infectious Diseases, vol. 10, no. 9, pp. 1665-1667, 2004.

[38] G. Valero, A. F. Cutrona, C. Watanakunakorn, and D. F. Talkington, "Group a streptococcus septicemia and an infected, ruptured abdominal aortic aneurysm associated with pharyngitis," Clinical Infectious Diseases, vol. 15, no. 3, pp. 525-527, 1992.

[39] T. M. Y. S. Sing, N. Young, I. C. O'Rourke, and P. Tomlinson, "Leaking mycotic abdominal aortic aneurysm," Australasian Radiology, vol. 38, no. 4, pp. 310-312, 1994.

[40] J. D. Bisognano, B. Young, J. M. Brown, E. A. Gill, F. C. Fang, and L. S. Zisman, "Diverse presentation of aberrant origin of the right subclavian artery: two case reports," Chest, vol. 112, no. 6, pp. 1693-1697, 1997.

[41] H. Barth, R. Moosdorf, J. Bauer, D. Schranz, and H. Akintürk, "Mycotic pseudoaneurysm of the aorta in children," Pediatric Cardiology, vol. 21, no. 3, pp. 263-266, 2000.

[42] S.-H. Chen, W.-C. Lin, C.-H. Lee, and W.-Y. Chou, "Spontaneous infective spondylitis and mycotic aneurysm: incidence, risk factors, outcome and management experience," European Spine Journal, vol. 17, no. 3, pp. 439-444, 2008.

[43] N. Vallejo, N. E. Picardo, P. Bourke et al., "The changing management of primary mycotic aortic aneurysms," Journal of Vascular Surgery, vol. 54, no. 2, pp. 334-340, 2011.

[44] L. Leiva, A. Arroyo, J. Porto, and R. Gesto, "Mycotic thoracoabdominal aortic aneurysm secondary to Streptococcus pyogenes: are we coming to the past?" Cirugía Española, vol. 85, no. 2, pp. 119-120, 2009 (Spanish).

[45] M. A. Hoffman, A. K. Ramey, J. Hammel, E. Truemper, S. Kutty, and A. Chatterjee, "Recurrent mycotic aneurysm in a 2-year-old boy with group a streptococcus bacteremia," Pediatric Infectious Disease Journal, vol. 31, no. 10, pp. 1080-1082, 2012. 


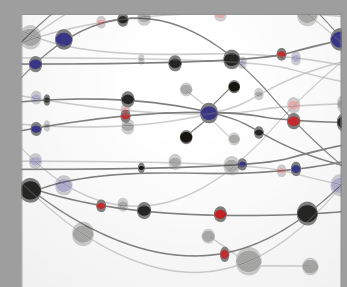

The Scientific World Journal
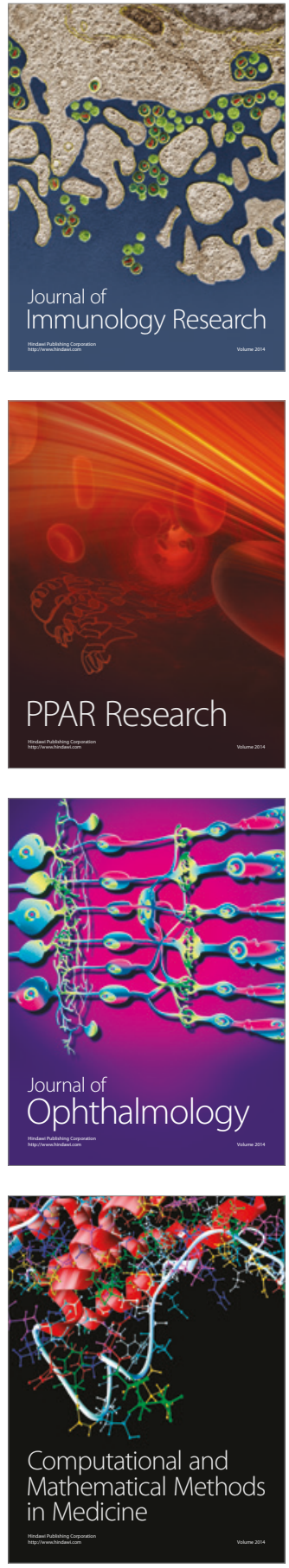

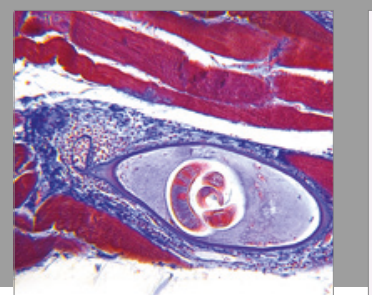

Gastroenterology Research and Practice
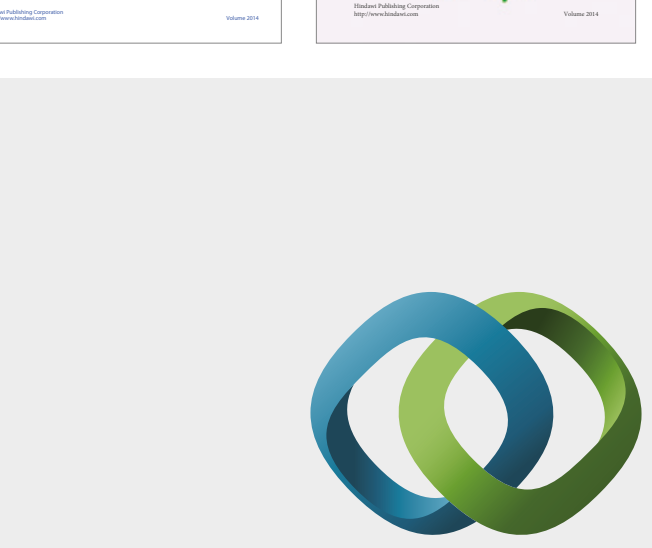

\section{Hindawi}

Submit your manuscripts at

https://www.hindawi.com
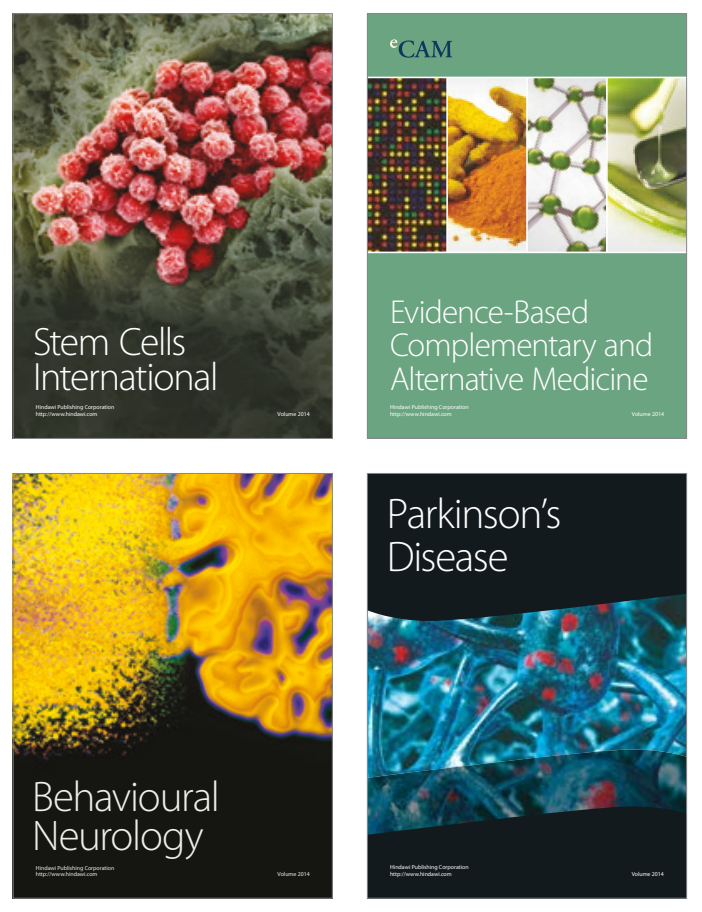
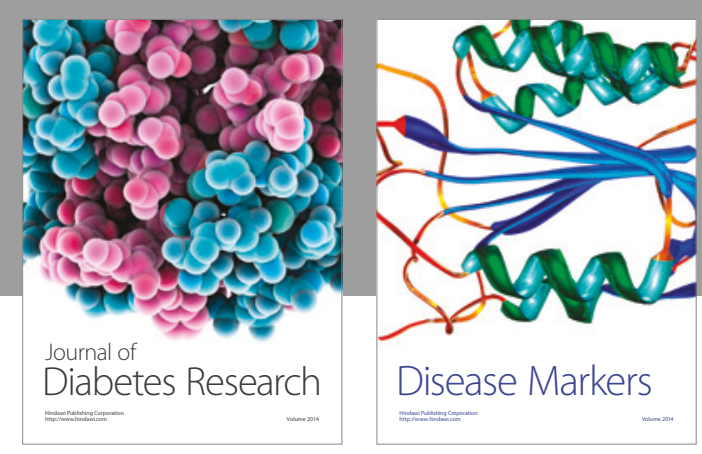

Disease Markers
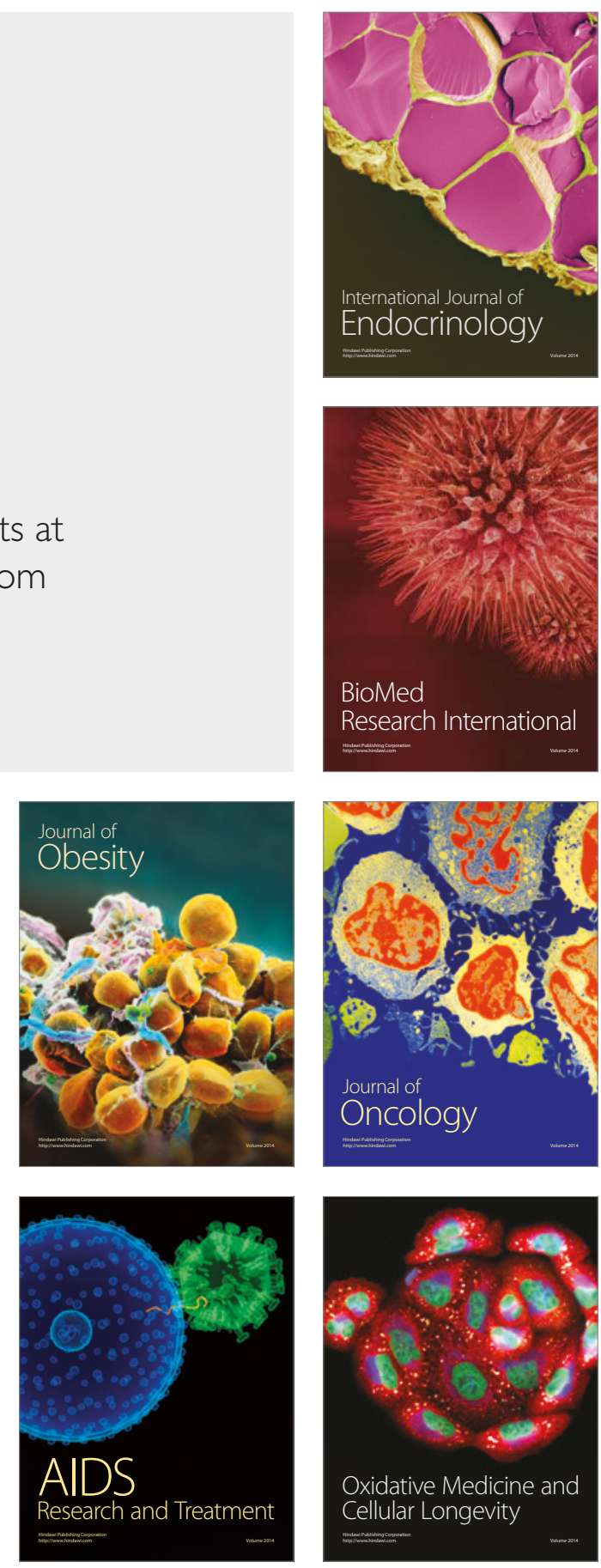